\title{
Predictive neutrino mass textures with origin of flavor symmetries
}

\author{
Tatsuo Kobayashi, ${ }^{1, *}$ Takaaki Nomura, ${ }^{2, \dagger}$ and Hiroshi Okada ${ }^{3, \$}$ \\ ${ }^{1}$ Department of Physics, Hokkaido University, Sapporo 060-0810, Japan \\ ${ }^{2}$ School of Physics, KIAS, Seoul 02455, Korea \\ ${ }^{3}$ Asia Pacific Center for Theoretical Physics, Pohang, Geyoengbuk 790-784, Republic of Korea
}

(Received 25 May 2018; published 19 September 2018)

\begin{abstract}
We investigate origins of predictive one-zero neutrino mass textures in a systematic way. Here, we search Abelian continuous(discrete) global symmetries, and non-Abelian discrete symmetries, and show how to realize these neutrino masses. We then propose a concrete model involving a dark matter candidate and an extra gauge boson and show their phenomenologies.
\end{abstract}

DOI: $10.1103 /$ PhysRevD.98.055025

\section{INTRODUCTION}

One of the most important issues in particle physics is to solve the mystery of the flavor structure of quarks and leptons, such as the generation number, mass hierarchy, mixing angles, and $\mathrm{CP}$ phases. Indeed, a huge number of studies have been done using various approaches. The texture ansatz is one of the interesting approaches. (See, for a review, e.g., [1].) By assuming a certain mass texture, one can derive several predictions among masses and mixing angles as well as $\mathrm{CP}$ phases.

The experimental data on the neutrino sector have become more precise through neutrino oscillation experiments, although there remain unknown aspects of the neutrino sector, e.g., the absolute values of neutrino masses and the question of whether neutrinos are Majorana or Dirac fermions. Thus, it would be interesting to apply the texture ansatz to the lepton sector. Actually, a lot of authors have historically been analyzing neutrino mass textures in various setups. For example, it is known that only seven neutrino mass patterns (two-zero textures) can predict neutrino oscillation data without conflict of current neutrino oscillation data [2] in the case where neutrinos are Majorana fermions with rank-three mass matrix [3].

Recently, type-I seesaw models with maximally restricted texture zeros have been systematically classified and analyzed numerically in Refs. [4,5], where charged-lepton mass matrix is assumed to be diagonal and only two

\footnotetext{
*kobayashi@particle.sci.hokudai.ac.jp

†nomura@kias.re.kr

*hiroshi.okada@apctp.org
}

Published by the American Physical Society under the terms of the Creative Commons Attribution 4.0 International license. Further distribution of this work must maintain attribution to the author(s) and the published article's title, journal citation, and DOI. Funded by SCOAP. families of right-handed neutrinos have Dirac mass terms with three active neutrinos. Then, the active neutrino mass matrix has one texture zero, and obviously one of active neutrinos is massless. Such patterns with one texture zero lead to several interesting predictions among neutrino masses and mixing angles. Indeed, such predictions for the normal hierarchy are not compatible with the experimental data. Also, some of patterns with one texture zero for the inverted hierarchy are already ruled out by experiments, while others are compatible.

Although the texture ansatz is quite interesting as mentioned above, it is unclear why such a pattern of mass matrix is realized. Our purpose is to explore origins of the neutrino mass textures obtained in Refs. [4,5]. In this paper, in order to realize those textures, we apply flavor symmetries such as global $U(1)$ symmetries, discrete Abelian symmetries $Z_{N}$, and non-Abelian discrete symmetries. The flavor symmetries provide a hint to explore the underlying theory beyond the standard model (SM).

Indeed non-Abelian discrete flavor symmetries have been studied by a lot of authors in order to realize the lepton masses and mixing angles as well as the $\mathrm{CP}$ phases. (See, for review, Refs. [6-8].) Furthermore, it has been shown that some non-Abelian discrete flavor symmetries appear in superstring theory with certain compactifications. Heterotic string theory on toroidal $Z_{N}$ orbifolds can lead non-Abelian flavor symmetries, e.g., $D_{4}$, and $\Delta(54)$ [9]. (See also $[10,11].)^{1}$ Similarly flavor symmetries can be realized in magnetized D-brane models and intersecting D-brane models within the framework of type II superstring theory $[13,14]$. In addition, these flavor symmetries may be subgroups of the modular symmetry in superstring theory [15]. Thus, flavor symmetry would make a bridge between the neutrino physics and underlying high energy physics.

${ }^{1}$ In Ref. [12], a relation between gauge symmetries and nonAbelian flavor symmetries is discussed. 
The minimal non-Abelian discrete symmetry is $S_{3}$ and the next one is $D_{4}$. Thus, in this paper we consider these $S_{3}$ and $D_{4}$ flavor symmetries as well as global $U(1)$ symmetry to realize the neutrino mass textures obtained in Refs. [4,5]. We will show that one can realize the desired textures by the $D_{4}$ flavor symmetry and $U(1)$ symmetry, but not by the $S_{3}$ flavor symmetries. Also it will be found that the $U(1)$ models need more Higgs fields than the $D_{4}$ flavor models. Then, we study the $D_{4}$ flavor model by using a concrete model. $^{2}$

This paper is organized as follows. In Sec. II, we give a brief review on the neutrino mass textures classified in Refs. [4,5]. In Sec. III, we study their realization by applying Abelian symmetries and non-Abelian discrete symmetries. In Sec. IV, we propose a concrete model, in which we formulate the boson sector, fermion sector, and dark matter sector (DM), and analyze collider physics based on an additional gauge symmetry. Then we discuss the DM candidate. Finally we conclude and discuss in Sec. V.

\section{NEUTRINO MASS TEXTURES}

In this section, we review the neutrino mass textures obtained in Refs. $[4,5]$. We consider the flavor basis, where charged lepton mass matrix is diagonal. Also we study the models, that only two families of right-handed neutrinos have Dirac mass terms with three families of left-handed neutrinos.

Active neutrino mass matrix is supposed to be induced from canonical mechanism; $m_{\nu} \approx m_{D} M_{N}^{-1} m_{D}^{T}$ after the spontaneously electroweak symmetry breaking. Here, $m_{D}$ is $(3 \times 2)$ Dirac mass matrix and $M_{N}$ is $(2 \times 2)$ Majorana mass matrix that come from the following Lagrangian; $y_{D_{i j}} \bar{L}_{L_{i}} \tilde{H}_{\mathrm{SM}} N_{R_{j}}+M_{N_{i j}} \bar{N}_{R_{i}}^{c} N_{R_{j}}$, where $\tilde{H}_{\mathrm{SM}} \equiv\left(i \sigma_{2}\right) H_{\mathrm{SM}}^{*}$ with the second Pauli matrix $\sigma_{2}, H_{\mathrm{SM}}$ is the SM Higgs, and $N_{R}$ are right-handed neutrinos. Then the neutrino mass matrix can be diagonalized by an unitary matrix $U_{\text {PMNS }}$ as

$$
U_{\mathrm{PMNS}}^{T} m_{\nu} U_{\mathrm{PMNS}}=\operatorname{diag}\left(m_{1}, m_{2}, m_{3}\right) \text {, }
$$

$$
U_{\mathrm{PMNS}}=\left[\begin{array}{ccc}
c_{12} c_{13} & s_{12} c_{13} & s_{13} e^{-i \delta} \\
-s_{12} c_{23}-c_{12} s_{23} s_{13} e^{i \delta} & c_{12} c_{23}-s_{12} s_{23} s_{13} e^{i \delta} & s_{23} c_{13} \\
s_{12} s_{23}-c_{12} c_{23} s_{13} e^{i \delta} & -c_{12} s_{23}-s_{12} c_{23} s_{13} e^{i \delta} & c_{23} c_{13}
\end{array}\right]\left[\begin{array}{ccc}
1 & 0 & 0 \\
0 & e^{i \alpha / 2} & 0 \\
0 & 0 & 1
\end{array}\right],
$$

where $m_{1,2,3}$ are neutrino mass eigenvalues, which are positive real, $c(s)_{12,13,23} \equiv \cos (\sin ) \theta_{12,13,23}$ are the three mixing angles, $\delta$ is the Dirac $C P$ phase, and $\alpha$ is the Majorana phase. Note here that there exists only one Majorana phase due to reduced $M_{N}$.

For the Dirac mass matrix $m_{D}$, the maximally allowed number of texture zeros is one or two. Then, such matrices $m_{D}$ are classified as [4]

$$
\begin{aligned}
& T_{1}:\left[\begin{array}{cc}
0 & \times \\
\times & 0 \\
\times & \times
\end{array}\right], \quad T_{2}:\left[\begin{array}{cc}
0 & \times \\
\times & \times \\
\times & 0
\end{array}\right], \quad T_{3}:\left[\begin{array}{cc}
\times & \times \\
0 & \times \\
\times & 0
\end{array}\right], \quad U_{1}:\left[\begin{array}{cc}
\times & \times \\
0 & \times \\
\times & \times
\end{array}\right], \quad U_{2}:\left[\begin{array}{cc}
\times & \times \\
\times & \times \\
0 & \times
\end{array}\right], \\
& T_{4}:\left[\begin{array}{cc}
\times & 0 \\
0 & \times \\
\times & \times
\end{array}\right], \quad T_{5}:\left[\begin{array}{cc}
\times & 0 \\
\times & \times \\
0 & \times
\end{array}\right], \quad T_{6}:\left[\begin{array}{cc}
\times & \times \\
\times & 0 \\
0 & \times
\end{array}\right], \quad U_{3}:\left[\begin{array}{cc}
\times & \times \\
\times & 0 \\
\times & \times
\end{array}\right], \quad U_{4}:\left[\begin{array}{cc}
\times & \times \\
\times & \times \\
\times & 0
\end{array}\right] \text {. }
\end{aligned}
$$

For the right-handed neutrino Majorana mass matrix $M_{N}$, the maximally allowed number of texture zeros is one or two. Then, such matrices $M_{N}$ are classified as [4]

$$
R_{1}:\left[\begin{array}{cc}
\times & 0 \\
0 & \times
\end{array}\right], \quad R_{2}:\left[\begin{array}{cc}
0 & \times \\
\times & \times
\end{array}\right], \quad R_{3}:\left[\begin{array}{cc}
\times & \times \\
\times & 0
\end{array}\right] \cdot S:\left[\begin{array}{cc}
0 & \times \\
\times & 0
\end{array}\right] .
$$

By combining these matrices, we can obtain the neutrino mass matrices $m_{\nu}$. Among all combinations, the realistic patterns of $m_{\nu}$ are classified [4]:

$$
a:\left[\begin{array}{ccc}
\times & 0 & \times \\
0 & \times & \times \\
\times & \times & \times
\end{array}\right], \quad b:\left[\begin{array}{ccc}
\times & \times & 0 \\
\times & \times & \times \\
0 & \times & \times
\end{array}\right], \quad c:\left[\begin{array}{ccc}
\times & \times & \times \\
\times & 0 & \times \\
\times & \times & \times
\end{array}\right], \quad d:\left[\begin{array}{ccc}
\times & \times & \times \\
\times & \times & \times \\
\times & \times & 0
\end{array}\right] .
$$

\footnotetext{
${ }^{2}$ See for models with the $D_{4}$ flavor symmetry, e.g., Refs. [16-21].
} 
TABLE I. Field contents of fermions and bosons and their charge assignments under $S U(2)_{L} \times U(1)_{Y} \times U(1)_{\mu-\tau}$ in the neutrino to realize the one-zero neutrino textures $T_{4}$, where $n_{1} \neq n_{2}, n_{1}, n_{2} \neq 0$ and $\left(n_{1}, n_{2} \pm 1, n_{1}+1\right) \neq \pm 1,+2$.

\begin{tabular}{|c|c|c|c|c|c|c|c|c|c|c|c|c|c|c|c|c|}
\hline Fields & $L_{L_{e}}$ & $L_{L_{\mu}}$ & $L_{L_{\tau}}$ & $e_{R}$ & $\mu_{R}$ & $\tau_{R}$ & $N_{R_{1}}$ & $N_{R_{2}}$ & $H_{\mathrm{SM}}$ & $H_{1}$ & $H_{2}$ & $H_{3}$ & $H_{4}$ & $\varphi_{1}$ & $\varphi_{2}$ & $\varphi_{3}$ \\
\hline$S U(2)_{L}$ & 2 & 2 & 2 & 1 & 1 & 1 & 1 & 1 & 2 & 2 & 2 & 2 & 2 & 1 & 1 & 1 \\
\hline$U(1)_{Y}$ & $-\frac{1}{2}$ & $-\frac{1}{2}$ & $-\frac{1}{2}$ & -1 & -1 & -1 & 0 & 0 & $\frac{1}{2}$ & $\frac{1}{2}$ & $\frac{1}{2}$ & $\frac{1}{2}$ & $\frac{1}{2}$ & 0 & 0 & 0 \\
\hline$U(1)_{\mu-\tau}$ & $0^{2}$ & $1^{2}$ & -1 & 0 & 1 & -1 & $n_{1}$ & $n_{2}$ & 0 & $n_{1}$ & $n_{2}-1$ & $n_{1}+1$ & $n_{2}+1$ & $-2 n_{1}$ & $-2 n_{2}$ & $-n_{1}-n_{2}$ \\
\hline
\end{tabular}

These are one-zero textures. Explicitly, these patterns are realized by the following combinations: $a$ for $\left(T_{1,4}, R_{1}\right), b$ for $\left(T_{2,5}, R_{1}\right), c$ for $\left(T_{3,4}, R_{2}\right)$ or $\left(T_{1,6}, R_{3}\right)$ or $\left(U_{1,3}, S\right)$ or $\left(U_{1}, R_{2}\right)$ or $\left(U_{3}, R_{3}\right)$, and $d$ for $\left(T_{5,6}, R_{2}\right)$ or $\left(T_{2,3}, R_{3}\right)$ or $\left(U_{2,4}, S\right)$ or $\left(U_{2}, R_{2}\right)$ or $\left(U_{4}, R_{3}\right)$. However, since all the combinations including $U$ require more Higgs doublets than those with $T_{1-6}$, we do not consider these cases. The other combinations lead to the neutrino mass matrix $m_{\nu}$, which is not compatible with the experimental data. Furthermore, all of the above patterns are compatible with the experiments for the inverted hierarchy, but not for the normal hierarchy. Also obviously, one of neutrinos is massless. For the above patterns of $m_{\nu}$, one finds the following relations [4]

$$
\begin{gathered}
\frac{m_{1}}{m_{2}}=-\frac{\left(U_{\mathrm{PMNS}}^{*}\right)_{i 2}\left(U_{\mathrm{PMNS}}^{*}\right)_{j 2}}{\left(U_{\mathrm{PMNS}}^{*}\right)_{i 1}\left(U_{\mathrm{PMNS}}^{*}\right)_{j 1}}, \\
\frac{1}{1+r_{\nu}}=\left|\frac{\left(U_{\mathrm{PMNS}}^{*}\right)_{i 2}\left(U_{\mathrm{PMNS}}^{*}\right)_{j 2}}{\left(U_{\mathrm{PMNS}}^{*}\right)_{i 1}\left(U_{\mathrm{PMNS}}^{*}\right)_{j 1}}\right|^{2}, \quad r_{\nu} \equiv \frac{\Delta m_{21}^{2}}{\Delta m_{31}^{2}},
\end{gathered}
$$

where we can identify $\Delta m_{21}^{2}+\left|\Delta m_{31}^{2}\right|=m_{2}^{2}$ and $\left|\Delta m_{31}^{2}\right|=$ $m_{1}^{2}$, since only inverted hierarchy is allowed for all the textures by the current neutrino oscillation data. Moreover, $\cos \delta$ can be written in terms of observables and $r_{\nu}$ by solving Eq. (2.6) directly, while $\cos \alpha$ is also obtained in terms of the same parameters of $\cos \delta$ by the fact that the imaginary part of Eq. (2.7) is zero. ${ }^{3}$

\section{REALIZATIONS OF TEXTURE ZEROS}

Here, we study realization by use of global $U(1)$ symmetry, $S_{3}$ and $D_{4}$ as well as $Z_{N}$.

\section{A. Abelian symmetries}

Here, we consider a global $U(1)$ symmetry to realize predictive textures, where we fix the number of righthanded neutrinos to be two generations, i.e., $N_{R_{1,2}}$. A flavor-dependent $U(1)$ symmetry in the lepton sector is useful to realize the diagonal mass matrix of the charged lepton sector. That is, the $U(1)_{\mu-\tau}, U(1)_{e-\mu}$ and $U(1)_{e-\tau}$ would be good candidates. Here, let us study the realization

\footnotetext{
${ }^{3}$ Neutrino mass eigenvalues are positive and real without loss of generality, because of reduced mass matrix.
}

of the Dirac mass texture $T_{4}$ by assuming the global $U(1)_{\mu-\tau}$ symmetry. ${ }^{4}$ The assignment of $U(1)_{\mu-\tau}$ charges is shown in Table I. We also assign $U(1)_{\mu-\tau}$ charges, $n_{1}$ and $n_{2}$ to $N_{R_{1}}$ and $N_{R_{2}}$. In order to realize Dirac neutrino mass terms, we have to introduce new $S U(2)_{L}$ doublet Higgs fields $H_{i}$, and their minimal number is four, i.e., $H_{i}(i=1$, $2,3,4)$. Also, in order to realize the mass matrix $M_{N}$, we have to introduce singlet scalar fields, $\varphi_{1,2,3}$. Here, the charges $n_{1}, n_{2}$ should satisfy the condition, $n_{1} \neq n_{2}$ and $n_{1}$, $n_{2} \neq 0$ in order to realize the desired Dirac texture of $T_{4}$, and they should also satisfy $\left(n_{1}, n_{2} \pm 1, n_{1}+1\right) \neq \pm 1,+2$ to forbid non-diagonal entries in the charged-lepton mass matrix. Under these symmetries and fields, one can write renormalizable coupling terms in the Lagrangian as follows:

$$
\begin{aligned}
-\mathcal{L}_{\text {Lepton }}= & \sum_{\ell=e, \mu, \tau} y_{\ell} \bar{L}_{L_{\ell}} H_{\mathrm{SM}} \ell_{R} \\
& +y_{D_{1}} \bar{L}_{L_{e}} \tilde{H}_{1} N_{R_{1}}+y_{D_{2}} \bar{L}_{L_{\mu}} \tilde{H}_{2} N_{R_{2}} \\
& +y_{D_{3}} \bar{L}_{L_{\tau}} \tilde{H}_{3} N_{R_{1}}+y_{D_{4}} \bar{L}_{L_{\tau}} \tilde{H}_{4} N_{R_{2}} \\
& +y_{N_{1}} \bar{N}_{R_{1}}^{C} N_{R_{1}} \varphi_{1}+y_{N_{2}} \bar{N}_{R_{2}}^{C} N_{R_{2}} \varphi_{2} \\
& +y_{N_{3}} \bar{N}_{R_{1}}^{C} N_{R_{2}} \varphi_{3}+\text { H.c. },
\end{aligned}
$$

where several dangerous Goldstone bosons (GBs) can be evaded by introducing soft-breaking mass terms under $U(1)_{\mu-\tau}$ symmetry; $m_{i j}^{2} H_{i}^{\dagger} H_{j}+$ H.c. $i \neq j=1-4$.

After the spontaneous symmetry breaking, the chargedlepton mass matrix and Dirac neutrino mass matrix are given by

$$
m_{\ell}=\frac{v_{H}}{\sqrt{2}}\left[\begin{array}{ccc}
y_{e} & 0 & 0 \\
0 & y_{\mu} & 0 \\
0 & 0 & y_{\tau}
\end{array}\right] \equiv\left[\begin{array}{ccc}
m_{e} & 0 & 0 \\
0 & m_{\mu} & 0 \\
0 & 0 & m_{\tau}
\end{array}\right]
$$

\footnotetext{
${ }^{4} \mathrm{~A}$ gauged symmetry will be analyzed in elsewhere, since several phenomenologies are very different from the global one. A comprehensive study has been done, e.g., by Ref. [22] in which two-zero textures are realized, imposing two flavor dependent $U(1)$ gauge symmetries.
} 
$m_{D}\left(T_{4}\right)=\frac{1}{\sqrt{2}}\left[\begin{array}{cc}y_{D_{1}} v_{H_{1}} & 0 \\ 0 & y_{D_{2}} v_{H_{2}} \\ y_{D_{2}} v_{H_{3}} & y_{D_{4}} v_{H_{4}}\end{array}\right] \equiv\left[\begin{array}{cc}m_{D_{1}} & 0 \\ 0 & m_{D_{2}} \\ m_{D_{3}} & m_{D_{4}}\end{array}\right]$,

where $v_{H}$ and $v_{H i}$ denote vacuum expectation values (VEVs) of the neutral components of $H_{\mathrm{SM}}$ and $H_{i}$, respectively. Then, the $T_{4}$ pattern of the Dirac neutrino mass matrix in Ref. [4] is derived. Also the right-handed neutrino mass matrix is given by

$$
M_{N}=\frac{1}{\sqrt{2}}\left[\begin{array}{ll}
y_{N_{1}} v_{\varphi_{1}} & y_{N_{3}} v_{\varphi_{3}} \\
y_{N_{3}} v_{\varphi_{3}} & y_{N_{2}} v_{\varphi_{2}}
\end{array}\right] \equiv\left[\begin{array}{cc}
M_{1} & M_{12} \\
M_{12} & M_{2}
\end{array}\right],
$$

where $v_{\varphi_{i}}$ denote VEVs of $\varphi_{i}$. From the above equation, one straightforwardly finds each of texture $R_{1}, R_{2}$, and $R_{3}$ in absence of $\varphi_{3}, \varphi_{1}$, and $\varphi_{2}$.

We can realize the Dirac neutrino mass texture $T_{1}$ with the same charge assignment except replacing the charges of $H_{1}$ and $H_{2}$ such that $H_{1}$ and $H_{2}$ have $U(1)_{\mu-\tau}$ charges, $n_{2}$ and $n_{1}-1$. Then, we can realize the Dirac neutrino mass,
$m_{D}\left(T_{1}\right)=\frac{1}{\sqrt{2}}\left[\begin{array}{cc}0 & y_{D_{1}} v_{H_{1}} \\ y_{D_{2}} v_{H_{2}} & 0 \\ y_{D_{2}} v_{H_{3}} & y_{D_{4}} v_{H_{4}}\end{array}\right] \equiv\left[\begin{array}{cc}0 & m_{D_{1}} \\ m_{D_{2}} & 0 \\ m_{D_{3}} & m_{D_{4}}\end{array}\right]$.

Similarly, the patterns, $T_{5}$ and $T_{2}$, are realized by $U(1)_{e-\mu}$ instead of $U(1)_{\mu-\tau}$. Also the patterns, $T_{6}$ and $T_{3}$, can be realized by use of $U(1)_{\tau-e}$ instead of $U(1)_{\mu-\tau}$.

Once any global $U(1)$ symmetries realize these predictive one-zero neutrino textures, discrete Abelian symmetries $Z_{N}$ are also possible in the same field contents, where $N \leq 19$.

\section{B. Non-Abelian discrete symmetries}

Here, we study the realization with non-Abelian discrete symmetries [7].

\section{1. $S_{3}$ symmetry}

First of all, we study the $S_{3}$ symmetry, which is the minimal group in the non-Abelian discrete symmetries. The irreducible representations of $S_{3}$ are the doublet 2, and the trivial singlet 1 and the nontrivial singlet $1^{\prime}$. Here, we use the real representation [7], ${ }^{5}$ and their products are expanded as

$$
\begin{gathered}
{\left[\begin{array}{l}
x_{1} \\
x_{2}
\end{array}\right]_{2} \otimes\left[\begin{array}{l}
y_{1} \\
y_{2}
\end{array}\right]_{2}=\left(x_{1} y_{1}+x_{2} y_{2}\right)_{1} \oplus\left(x_{1} y_{2}-x_{2} y_{1}\right)_{1^{\prime}} \oplus\left[\begin{array}{l}
x_{1} y_{2}+x_{2} y_{1} \\
x_{1} y_{1}-x_{2} y_{2}
\end{array}\right]_{2},} \\
{\left[\begin{array}{l}
x_{1} \\
x_{2}
\end{array}\right]_{2} \otimes\left(y^{\prime}\right)_{1^{\prime}}=\left[\begin{array}{c}
-x_{2} y^{\prime} \\
x_{1} y^{\prime}
\end{array}\right]_{2}, \quad(x)_{1^{\prime}} \otimes(y)_{1^{\prime}}=(x y)_{1} .}
\end{gathered}
$$

We assign $\left(L_{L_{\ell}}, \ell_{R}\right)(\ell=e, \mu)$ to the $S_{3}$ doublets 2 , and $L_{L_{\tau}}, \tau_{R}$ to the $S_{3}$ trivial singlets 1 . In addition, we introduce four Higgs fields, which correspond to the $S_{3}$ doublet, $H_{D} \sim 2, S_{3}$ singlets, $H_{1} \sim 1$, and $H_{2} \sim 1^{\prime}$. Then the renormalizable coupling terms of the charged-lepton sector are given by

$$
\begin{aligned}
\mathcal{L}_{\ell}= & y_{\ell_{1}}\left[\left(\bar{L}_{L_{e}} H_{D_{2}}+\bar{L}_{L_{\mu}} H_{D_{1}}\right) e_{R}+\left(\bar{L}_{L_{e}} H_{D_{1}}-\bar{L}_{L_{\mu}} H_{D_{2}}\right) \mu_{R}\right] \\
& +y_{\ell_{2}}\left(\bar{L}_{L_{e}} H_{D_{1}}+\bar{L}_{L_{\mu}} H_{D_{2}}\right) \tau_{R}+y_{\ell_{3}}\left(\bar{L}_{L_{e}} H_{1} e_{R}+\bar{L}_{L_{\mu}} H_{1} \mu_{R}\right)+y_{\ell_{4}} \bar{L}_{L_{\tau}}\left(H_{D_{1}} e_{R}+H_{D_{2}} \mu_{R}\right) \\
& +y_{\ell_{5}} \bar{L}_{L_{\tau}} H_{1} \tau_{R}+y_{\ell_{6}}\left(\bar{L}_{L_{e}} H_{2} \mu_{R}-\bar{L}_{L_{\mu}} H_{2} e_{R}\right)+\text { H.c. }
\end{aligned}
$$

After the spontaneously electroweak symmetry breaking, the charged-lepton mass matrix can be found as

$$
m_{\ell}=\frac{1}{\sqrt{2}}\left[\begin{array}{ccc}
y_{\ell_{1}} v_{D_{1}}+y_{\ell_{3}} v_{1} & y_{\ell_{1}} v_{D_{1}}+y_{\ell_{6}} v_{2} & y_{\ell_{2}} v_{D_{1}} \\
y_{\ell_{1}} v_{D_{2}}-y_{\ell_{6}} v_{2} & -y_{\ell_{1}} v_{D_{2}}+y_{\ell_{3}} v_{1} & y_{\ell_{2}} v_{D_{2}} \\
y_{\ell_{4}} v_{D_{1}} & y_{\ell_{4}} v_{D_{2}} & y_{\ell_{5}} v_{1}
\end{array}\right]
$$

where VEVs are denoted by $\left\langle H_{i}\right\rangle \equiv v_{i} / \sqrt{2}$ and $\left\langle H_{D_{i}}\right\rangle \equiv v_{D_{i}} / \sqrt{2}$ for $i=1$, 2. Once $\left\langle H_{D}\right\rangle=\left\langle H_{2}\right\rangle=0$, the diagonal charged-lepton mass matrix is realized;

\footnotetext{
${ }^{5}$ Note here that the complex representations cannot construct the diagonal mass matrix of charged lepton.
} 


$$
m_{\ell}=\frac{1}{\sqrt{2}}\left[\begin{array}{ccc}
y_{\ell_{3}} v_{1} & 0 & 0 \\
0 & y_{\ell_{3}} v_{1} & 0 \\
0 & 0 & y_{\ell_{5}} v_{1}
\end{array}\right]
$$

However, from the above mass matrix, one cannot reproduce the mass difference between the masses of electron and muon. Thus, $S_{3}$ symmetry is not favorable. ${ }^{6}$

\section{2. $D_{4}$ symmetry}

Next, we investigate the $D_{4}$ flavor symmetry that is the next minimal group in the non-Abelian discrete symmetries. The irreducible representations of $D_{4}$ symmetry are the doublet 2 , and the trivial singlet 1 , and three nontrivial singlets, $1^{\prime}, 1^{\prime \prime}, 1^{\prime \prime \prime}{ }^{7}$ Here, we also use the real representation, and their productions are shown in the Appendix. We assign $\left(L_{L_{\ell}}, \ell_{R}\right)$ $(\ell=e, \mu)$ to the $D_{4}$ doublets 2 , and $L_{L_{\tau}}, \tau_{R}$ to the $D_{4}$ trivial singlets 1 . In addition, we introduce 6 Higgs fields, which correspond to all of the $D_{4}$ irreducible representations, 2, 1, $1^{\prime}, 1^{\prime}, 1^{\prime \prime}, 1^{\prime \prime \prime}$, that is, $H_{D} \sim 2$, $H_{1} \sim 1, H_{2} \sim 1^{\prime}, H_{3} \sim 1^{\prime \prime}, H_{4} \sim 1^{\prime \prime \prime}$. Then the renormalizable coupling terms of the charged-lepton sector are given by

$$
\begin{aligned}
\mathcal{L}_{\ell} & =y_{\ell_{1}}\left(\bar{L}_{L_{e}} H_{D_{1}}+\bar{L}_{L_{\mu}} H_{D_{2}}\right) \tau_{R}+y_{\ell_{2}} \bar{L}_{L_{\tau}}\left(H_{D_{1}} e_{R}+H_{D_{2}} \mu_{R}\right) \\
& +y_{\ell_{3}}\left(\bar{L}_{L_{e}} H_{1} e_{R}+\bar{L}_{L_{\mu}} H_{1} \mu_{R}\right)+y_{\ell_{4}}\left(\bar{L}_{L_{e}} H_{2} e_{R}-\bar{L}_{L_{\mu}} H_{2} \mu_{R}\right) \\
& +y_{\ell_{5}}\left(\bar{L}_{L_{e}} H_{3} \mu_{R}+\bar{L}_{L_{\mu}} H_{3} e_{R}\right)+y_{\ell_{6}}\left(\bar{L}_{L_{e}} H_{4} \mu_{R}-\bar{L}_{L_{\mu}} H_{4} e_{R}\right) \\
& +y_{\ell_{7}} \bar{L}_{L_{\tau}} H_{1} \tau_{R}+\text { H.c. }
\end{aligned}
$$

After the spontaneously electroweak symmetry breaking, the charged-lepton mass matrix can be found as

$m_{\ell}=\frac{1}{\sqrt{2}}\left[\begin{array}{ccc}y_{\ell_{3}} v_{1}+y_{\ell_{4}} v_{2} & y_{\ell_{5}} v_{3}+y_{\ell_{6}} v_{4} & y_{\ell_{1}} v_{D_{1}} \\ y_{\ell_{5}} v_{3}-y_{\ell_{6}} v_{4} & y_{\ell_{3}} v_{1}-y_{\ell_{4}} v_{2} & y_{\ell_{1}} v_{D_{2}} \\ y_{\ell_{2}} v_{D_{1}} & y_{\ell_{2}} v_{D_{2}} & y_{\ell_{7}} v_{1}\end{array}\right]$,

where their VEVs are denoted by $\left\langle H_{i}\right\rangle \equiv v_{i} / \sqrt{2}$ $(i=1, \ldots, 4)$ and $\left\langle H_{D_{i}}\right\rangle \equiv v_{D_{j}} / \sqrt{2}$ for $j=1,2$. Once $\left\langle H_{D}\right\rangle=\left\langle H_{2,3,4}\right\rangle=0$ and/or $y_{\ell_{1,2,5}}=0$, the diagonal charged-lepton mass matrix is realized;

\footnotetext{
${ }^{6}$ Note here that Refs. $[23,24]$ realize the appropriate chargedlepton mass matrix, by imposing an additional $Z_{2}$ symmetry.

${ }^{7}$ The singlets, $1,1^{\prime}, 1^{\prime \prime}, 1^{\prime \prime \prime}$, correspond to $1_{++}, 1_{--}, 1_{+-}, 1_{-+}$ in Ref. [7], respectively.
}

$m_{\ell}=\frac{1}{\sqrt{2}}\left[\begin{array}{ccc}y_{\ell_{3}} v_{1}+y_{\ell_{4}} v_{2} & 0 & 0 \\ 0 & y_{\ell_{3}} v_{1}-y_{\ell_{4}} v_{2} & 0 \\ 0 & 0 & y_{\ell_{7}} v_{1}\end{array}\right]$.

From the above equation, one can reproduce the mass difference between the masses of electron and muon. Thus, the $D_{4}$ flavor symmetry can be the minimal candidate to reproduce the desired textures. To realize the diagonal mass matrix of the charged lepton sector, we just need $H_{1}$ and $H_{2}$, but we do not need $H_{D}$ or $H_{3,4}$.

Next, let us explore the neutrino sector; Dirac and Majorana masses. We classify the models by assigning systematically two right-handed neutrinos to two of the $D_{4}$ irreducible representations, $2,1,1^{\prime}, 1^{\prime \prime}, 1^{\prime \prime \prime}$.

In the case of $\left(N_{R_{1}}, N_{R_{2}}\right) \sim 2$.- The Majorana mass matrix is given by

$$
M_{N}=M\left[\begin{array}{ll}
1 & 0 \\
0 & 1
\end{array}\right]
$$

where these two masses are degenerated. Then the Dirac neutrino mass matrix is given by

$$
m_{D}=\frac{1}{\sqrt{2}}\left[\begin{array}{cc}
y_{D_{1}} v_{1}+y_{D_{2}} v_{2} & y_{D_{3}} v_{3}+y_{D_{4}} v_{4} \\
y_{D_{3}} v_{3}-y_{D_{4}} v_{4} & y_{D_{1}} v_{1}-y_{D_{2}} v_{2} \\
y_{D_{5}} v_{D_{1}} & y_{D_{5}} v_{D_{2}}
\end{array}\right] .
$$

Hence one finds the desired Dirac mass matrix in the case of $\left\langle H_{3,4}\right\rangle=0^{8}$

$$
m_{D}\left(T_{4}\right)=\left[\begin{array}{cc}
m_{D_{1}} & 0 \\
0 & m_{D_{2}} \\
m_{D_{3}} & m_{D_{4}}
\end{array}\right]
$$

For this realization, we need $H_{D}, H_{1}$ and $H_{2}$, but not $H_{3}$ or $\mathrm{H}_{4}$.

Now, let us study the models, that $N_{R_{1}}$ and $N_{R_{2}}$ are assigned to two $D_{4}$ singlets. If one assigns $N_{R_{1}}$ and $N_{R_{2}}$ into the same singlet representation under $D_{4}$, the Majorana mass matrix does not give any vanishing elements without imposing additional symmetries. Thus, we restrict ourselves to the models such that $N_{R_{1}}$ and $N_{R_{2}}$ are assigned to $D_{4}$ singlets different from each other.

When we assign $N_{R_{1}}$ and $N_{R_{2}}$ into different $D_{4}$ singlets such as $\left(N_{R_{1}}, N_{R_{2}}\right) \sim\left(1,1^{\prime}\right),\left(1^{\prime \prime}, 1^{\prime \prime \prime}\right)$, etc., the Majorana mass matrix is give by

\footnotetext{
${ }^{8}$ In case of $\left\langle H_{1,2}\right\rangle=0, T_{1}$ can be obtained. However, the electron and muon are massless. Thus, this case is ruled out.
} 
TABLE II. Field contents of fermions and bosons and their charge assignments under $S U(2)_{L} \times U(1)_{Y} \times U(1)_{B-L} \times D_{4}$ in the neutrino and Higgs sector, where $\ell=e, \mu$ is flavor index.

\begin{tabular}{|c|c|c|c|c|c|c|c|c|c|c|c|c|c|c|c|c|}
\hline Fields & $L_{L_{\ell}}$ & $L_{L_{\tau}}$ & $\ell_{R}$ & $\tau_{R}$ & $N_{R_{i}}$ & $N_{R_{\tau}}$ & $H$ & $H_{2}$ & $\eta_{1}$ & $\eta_{1^{\prime}}$ & $\eta_{D}$ & $\varphi_{8}$ & $\varphi_{8}^{\prime}$ & $\varphi_{10}$ & $\zeta$ & $\varphi_{2}$ \\
\hline$S U(2)_{L}$ & 2 & 2 & 1 & 1 & 1 & 1 & 2 & 2 & 2 & 2 & 2 & 1 & 1 & 1 & 2 & 1 \\
\hline$U(1)_{Y}$ & $-\frac{1}{2}$ & $-\frac{1}{2}$ & -1 & -1 & 0 & 0 & $\frac{1}{2}$ & $\frac{1}{2}$ & $\frac{1}{2}$ & $\frac{1}{2}$ & $\frac{1}{2}$ & 0 & 0 & 0 & $\frac{1}{2}$ & 0 \\
\hline$U(1)_{B-L}$ & -1 & -1 & -1 & -1 & -4 & 5 & 0 & 0 & -3 & -3 & -3 & 8 & 8 & 10 & -6 & 2 \\
\hline$D_{4}$ & 2 & 1 & 2 & 1 & 2 & 1 & 1 & $1^{\prime}$ & 1 & $1^{\prime}$ & 2 & 1 & $1^{\prime}$ & 2 & 1 & 1 \\
\hline
\end{tabular}

$$
M_{N}=\left[\begin{array}{cc}
M_{1} & 0 \\
0 & M_{2}
\end{array}\right]
$$

That is the $R_{1}$ form.

In the case of $\left(N_{R_{1}}, N_{R_{2}}\right) \sim\left(1,1^{\prime}\right)$.- The Dirac neutrino Yukawa mass matrix is given by

$$
m_{D}=\frac{1}{\sqrt{2}}\left[\begin{array}{cc}
y_{D_{1}} v_{D_{1}} & y_{D_{2}} v_{D_{1}} \\
y_{D_{1}} v_{D_{2}} & -y_{D_{2}} v_{D_{2}} \\
y_{D_{3}} v_{1} & y_{D_{4}} v_{2}
\end{array}\right] \text {. }
$$

This form cannot clearly reproduce any types of desired Dirac mass matrices, since $y_{D_{1}}$ and $y_{D_{2}}$ are located in the same column of upper $(2 \times 2)$ matrix. When we assign $\left(N_{R_{1}}, N_{R_{2}}\right) \sim\left(1^{\prime \prime}, 1^{\prime \prime \prime}\right)$, we obtain a similar result. Then, these two cases are not favorable, but the other cases are favorable.

In the case of $\left(N_{R_{1}}, N_{R_{2}}\right) \sim\left(1\left(1^{\prime}\right), 1^{\prime \prime}\left(1^{\prime \prime \prime}\right)\right)$. - The Dirac neutrino mass matrix is given by

$$
m_{D}=\frac{1}{\sqrt{2}}\left[\begin{array}{cc}
y_{D_{1}} v_{D_{1}} & y_{D_{2}} v_{D_{2}} \\
\pm y_{D_{1}} v_{D_{2}} & \pm y_{D_{1}} v_{D_{1}} \\
y_{D_{3}} v_{1} & y_{D_{4}} v_{3(4)}
\end{array}\right],
$$

where "+" and "-" in the $(2,1)$ component corresponds to $N_{R_{1}} \sim 1$ and $N_{R_{1}} \sim 1^{\prime}$, respectively, and "+" or "-" in the $(2,2)$ component and $v_{3}$ and $v_{4}$ in the $(3,3)$ component correspond to $N_{R_{2}} \sim 1^{\prime \prime}$ and $N_{R_{2}} \sim 1^{\prime \prime \prime}$, respectively. One straightforwardly finds the desired Dirac mass matrices $T_{1}$ and $T_{4}$ in the cases with $\left\langle H_{D_{1}}\right\rangle=0$ and $\left\langle H_{D_{2}}\right\rangle=0$, respectively. For example, in the case of $\left(N_{R_{1}}, N_{R_{2}}\right) \sim$ $\left(1,1^{\prime \prime}\right)$, we need $H_{D}, H_{1,2,3}$, but not $H_{4}$.

In order to obtain $T_{2,3,5,6}$, one straightforwardly finds them by reassigning the fields of the SM leptons. For example, once we assign $\left(L_{L_{e}}, L_{L_{\tau}}\right) \sim\left(e_{R}, \tau_{R}\right) \sim 2$, and $\left(L_{L_{\mu}}, \mu_{R}\right) \sim 1$, then one finds $T_{2}$ or $T_{5}$. On the other hand, when we assign $\left(L_{L_{\mu}}, L_{L_{\tau}}\right) \sim\left(\mu_{R}, \tau_{R}\right) \sim 2$, and $\left(L_{L_{e}}, e_{R}\right) \sim 1$, then one finds $T_{3}$ or $T_{6}$.

To summarize results in this section, one can realize the desired textures by $D_{4}$, but not by $S_{3}$. Indeed, the $D_{4}$ flavor symmetry is interesting from the viewpoints of both high energy physic [9-11,13-15,25-27]. and bottom-up model building approach [16-21]. Similarly, we can discuss realization by using other non-Abelian discrete flavor symmetries. Also we can realize the desired textures by Abelian symmetries, $U(1)$ and $Z_{N}$. We need more Higgs fields in the Abelian models than the $D_{4}$ models. Thus, the $D_{4}$ flavor symmetry is useful to realize the desired textures. Note here that the textures $c$ and $d$ in Eq. (2.5) cannot be realized by $D_{4}$ symmetry, because $M_{N}$ is diagonal $\left(R_{1}\right.$ form). ${ }^{9}$ In the next section, we propose a concrete model with the $D_{4}$ flavor symmetry.

\section{A CONCRETE MODEL IN $D_{4}$ SYMMETRY}

Here, we study a concrete model based on the $D_{4}$ symmetry. First, we explain our setup. Basically, our model corresponds to the scenario, where $N_{R_{1,2}}$ are assigned to the $D_{4}$ doublet in Sec. III B 2. In addition, we also introduce the third right-handed neutrino $N_{R_{3}}$, but arrange it such that $N_{R_{3}}$ has no Dirac mass term with left-handed neutrino and no Majorana mass terms with $N_{R_{1,2}}$. For such a purpose, we assume additional $U(1)$ gauge symmetry, that is, $U(1)_{B-L}$. Its charge assignment is the same as the conventional one except the right-handed neutrino sector. For the righthanded neutrino sector, we assign $U(1)_{B-L}$ charges, -4 , $-4,5$ to $N_{R_{1,2,3}}$, respectively. That is the so-called alternative $U(1)_{B-L}$ [28-34]. All gauge anomalies are canceled with this choice. In the boson sector, we introduce several new bosons $H_{2}, \eta_{1,1^{\prime}, D}, \varphi_{2,8}, \varphi_{8}^{\prime}, \zeta$ in addition to the SM Higgs $H$, where $H$ gives the masses for the quark sector and the charged lepton sector, while $\mathrm{H}_{2}$ gives mass difference between electron(positron) and muon(antimuon). Here, their VEVs are symbolized by $\langle H\rangle \equiv v_{H},\left\langle H_{2}\right\rangle \equiv v_{H}^{\prime}$, $\left\langle\eta_{1,1^{\prime}, D}\right\rangle \equiv v_{\eta, \eta^{\prime} \eta_{D}},\left\langle\varphi_{2,8}\right\rangle \equiv v_{\varphi_{2}, \varphi_{8}},\left\langle\varphi_{8}^{\prime}\right\rangle \equiv v_{\varphi_{8}^{\prime}},\langle\zeta\rangle \equiv v_{\zeta}$. Also $\eta$ and $\varphi_{8}$, respectively, provide the Dirac and righthanded neutrino masses, $\eta^{\prime}$ and $\varphi_{8}^{\prime}$, respectively, provide the difference between the (1-1) and (2-2) elements of $m_{D}$ and $M_{N}$, and $\eta_{D}$ gives the masses for the third row of Dirac mass matrix. $\zeta$ and $\varphi_{2}$ play a role in evading dangerous GBs due to accidental symmetries in the scalar potential. The $D_{4}$ symmetry assures diagonal mass matrices for charged

\footnotetext{
${ }^{9}$ If an additional symmetry is introduced in the basis of $\left(N_{R_{1}}, N_{R_{2}}\right) \sim 1$ under $D_{4}$ symmetry, $c$ and $d$ can be realized, but this is beyond our scope.
} 
leptons and right-handed neutrinos, and $U(1)_{B-L}$ plays a role in restricting $(2 \times 2)$ mass matrix for right-handed neutrinos which contribute to active neutrino masses. In addition, our $U(1)_{B-L}$ charge assignment makes $N_{R_{3}}$ stable and it can be a DM candidate. All the field contents and their charge assignments are shown in Table II. Under these contents with symmetries, one can write renormalizable Yukawa coupling terms and the Higgs potential as follows ${ }^{10}$ :

$$
\begin{aligned}
-\mathcal{L}_{\text {Lepton }}= & y_{\ell}\left(\bar{L}_{L_{e}} e_{R}+\bar{L}_{L_{\mu}} \mu_{R}\right) H+y_{\ell}^{\prime}\left(\bar{L}_{L_{e}} e_{R}-\bar{L}_{L_{\mu}} \mu_{R}\right) H_{2}+y_{\tau} \bar{L}_{L_{\tau}} \tau_{R} H+y_{D}\left(\bar{L}_{L_{e}} N_{R_{e}}+\bar{L}_{L_{\mu}} N_{R_{\mu}}\right) \tilde{\eta}_{1} \\
& +y_{D}^{\prime}\left(\bar{L}_{L_{e}} N_{R_{e}}-\bar{L}_{L_{\mu}} N_{R_{\mu}}\right) \tilde{\eta}_{1}^{\prime}+y_{D_{3}} \bar{L}_{L_{\tau}}\left(N_{R_{e}} \tilde{\eta}_{D_{1}}+N_{R_{\mu}} \tilde{\eta}_{D_{2}}\right)+y_{N}\left(\bar{N}_{R_{e}}^{C} N_{R_{e}}+\bar{N}_{R_{\mu}}^{C} N_{R_{\mu}}\right) \varphi_{8} \\
& +y_{N}^{\prime}\left(\bar{N}_{R_{e}}^{C} N_{R_{e}}-\bar{N}_{R_{\mu}}^{C} N_{R_{\mu}}\right) \varphi_{8}^{\prime}+\text { H.c. },
\end{aligned}
$$

$$
\begin{aligned}
V= & \lambda_{1,1^{\prime}, D}\left(\zeta^{\dagger} \eta_{1,1^{\prime}, D}\right)\left(H^{\dagger} \eta_{1,1^{\prime}, D}\right)+\lambda_{1}^{\prime}\left(\zeta^{\dagger} \eta_{1}\right)\left(H_{2}^{\dagger} \eta_{1^{\prime}}\right) \\
& +\lambda_{D}^{\prime}\left(\zeta^{\dagger} \eta_{D}\right)\left(H_{2}^{\dagger} \eta_{D}\right)+\lambda_{0}\left(\zeta^{\dagger} H\right) \varphi_{8}^{*} \varphi_{2}+\text { H.c. },
\end{aligned}
$$

where $V$ is the Higgs potential with non-trivial terms. These nontrivial terms forbid dangerous GBs arising from isospin doublets that spoil the model. In our model, we have two GBs that can be identified with $C P$-odd bosons of $\varphi_{2}$ and $\varphi_{8}\left(\varphi_{8}^{\prime}\right) .{ }^{11}$

\section{A. Lepton sector}

The resulting mass matrices are give by

$$
\begin{aligned}
& m_{\ell}=\frac{1}{\sqrt{2}}\left[\begin{array}{ccc}
y_{\ell} v_{H}+y_{\ell}^{\prime} v_{H}^{\prime} & 0 & 0 \\
0 & y_{\ell} v_{H}-y_{\ell}^{\prime} v_{H}^{\prime} & 0 \\
0 & 0 & y_{\tau} v_{H}
\end{array}\right] \\
& \equiv\left[\begin{array}{ccc}
m_{e} & 0 & 0 \\
0 & m_{\mu} & 0 \\
0 & 0 & m_{\tau}
\end{array}\right] \text {, } \\
& m_{D}=\frac{1}{\sqrt{2}}\left[\begin{array}{cc}
y_{D} v_{\eta}+y_{D}^{\prime} v_{\eta^{\prime}} & 0 \\
0 & y_{D} v_{\eta}-y_{D}^{\prime} v_{\eta^{\prime}} \\
y_{D_{3}} v_{\eta_{D_{1}}} & y_{D_{3}} v_{\eta_{D_{2}}^{\prime}}^{\prime}
\end{array}\right] \\
& \equiv\left[\begin{array}{cc}
m_{D_{1}} & 0 \\
0 & m_{D_{2}} \\
m_{D_{3}} & m_{D_{4}}
\end{array}\right] \\
& M_{N}=\frac{1}{\sqrt{2}}\left[\begin{array}{cc}
y_{N} v_{\varphi_{8}}+y_{N}^{\prime} v_{\varphi_{8}^{\prime}} & 0 \\
0 & y_{N} v_{\varphi_{8}}-y_{N}^{\prime} v_{\varphi_{8}^{\prime}}
\end{array}\right] \\
& \equiv\left[\begin{array}{cc}
M_{1} & 0 \\
0 & M_{2}
\end{array}\right] \text {. }
\end{aligned}
$$

\footnotetext{
${ }^{10}$ We show valid multiplication rules for $D_{4}$ in the Appendix.

${ }^{11}$ In addition, one has to introduce soft breaking terms of $D_{4}$ symmetry in order to forbid accidental symmetries that also induce dangerous GBs. The breaking patterns are given by Ref. [7], and any patterns are fine because it does not affect our model. Thus, we do not discuss this issue further.
}

The above neutrino Dirac mass matrix $m_{D}$ corresponds to Eq. (3.17). Also the above Majorana mass matrix $M_{N}$ basically corresponds to Eq. (3.15). However, since there are two fields $\varphi_{8}$ and $\varphi_{8}^{\prime}$, we obtain $M_{1} \neq M_{2}$. Then, we can obtain

$$
m_{\nu} \approx\left[\begin{array}{ccc}
\frac{m_{D_{1}}^{2}}{M_{1}} & 0 & \frac{m_{D_{1}} m_{D_{3}}}{M_{1}} \\
0 & \frac{m_{D_{2}}^{2}}{M_{2}} & \frac{m_{D_{2}} m_{D_{4}}}{M_{2}} \\
\frac{m_{D_{1}} m_{D_{3}}}{M_{1}} & \frac{m_{D_{2}} m_{D_{4}}}{M_{2}} & \frac{m_{D_{3}}^{2}}{M_{1}}+\frac{m_{D_{4}}^{2}}{M_{2}}
\end{array}\right],
$$

which corresponds to the pattern $a$ in Eq. (2.5). Applying the discussion in Sec. II to our model, we find

$$
\frac{m_{1}}{m_{2}}=-\frac{\left(U_{\mathrm{PMNS}}^{*}\right)_{12}\left(U_{\mathrm{PMNS}}^{*}\right)_{22}}{\left(U_{\mathrm{PMNS}}^{*}\right)_{11}\left(U_{\mathrm{PMNS}}^{*}\right)_{21}}
$$

Therefore, one obtains two relations from the above relation:

$$
\begin{gathered}
\cos \delta=\frac{\left[s_{12}^{4}\left(1+r_{\nu}\right)-c_{12}^{4}\right] s_{23}^{2} s_{13}^{2}+r_{\nu} c_{23}^{2} s_{12}^{2} c_{12}^{2}}{2\left[s_{12}^{2}\left(1+r_{\nu}\right)+c_{12}^{2}\right] s_{12} c_{12} s_{23} c_{23} s_{13}}, \\
\cos \alpha=\frac{-\left[s_{12}^{4}\left(1+r_{\nu}\right)+c_{12}^{4}\right] s_{23}^{2} s_{13}^{2}+\left(2+r_{\nu}\right) c_{23}^{2} s_{12}^{2} c_{12}^{2}}{2 \sqrt{\left(1+r_{\nu}\right.}\left(c_{23}^{2}+s_{23}^{2} s_{13}^{2}\right) s_{12}^{2} c_{12}^{2}},
\end{gathered}
$$

where Eq. (4.8) is derived by solving Eq. (4.7) directly, while Eq. (4.9) is obtained by the fact that the imaginary part of Eq. (4.7) is vanishing. Applying the current neutrino oscillation data [2], we find some predictions. In Fig. 1, we show the allowed region between $\alpha / \pi$ and $\delta / \pi$ and it suggests as follows; $0.075 \lesssim \alpha / \pi \lesssim 0.15$ and $0.49 \lesssim \delta / \pi \lesssim$ 0.52 at $3 \sigma$ confidential level (CL) (blue region), $0.105 \lesssim$ $\alpha / \pi \lesssim 0.13$ and $0.50 \lesssim \delta / \pi \lesssim 0.51$ at $1 \sigma \mathrm{CL}$ (red region), and $(\alpha / \pi, \delta / \pi) \approx(0.11,0.51)$ at best-fit value (BF) (black dot).

Consistency check: Replacing $a \equiv \frac{m_{D_{1}}^{2}}{M_{1}}, b \equiv \frac{m_{D_{2}}^{2}}{M_{2}}, r_{31} \equiv$ $m_{D_{3}} / m_{D_{1}}, r_{42} \equiv m_{D_{4}} / m_{D_{2}}$ Eq. (4.6) can be rewritten in terms of four parameters as follows: 


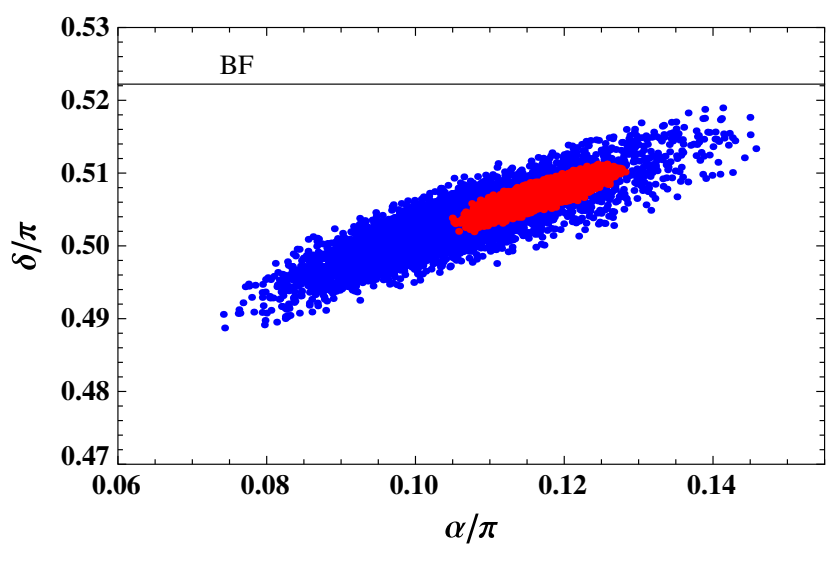

FIG. 1. Allowed region between $\alpha / \pi$ and $\delta / \pi$ to satisfy the current neutrino oscillation data. Also the blue, red, and black regions, respectively, represent predictions in light of the experimental input results at $3 \sigma \mathrm{CL}, 1 \sigma \mathrm{CL}$, and BF. Here, the black horizontal line presents the best-fit value $(\mathrm{BF})$.

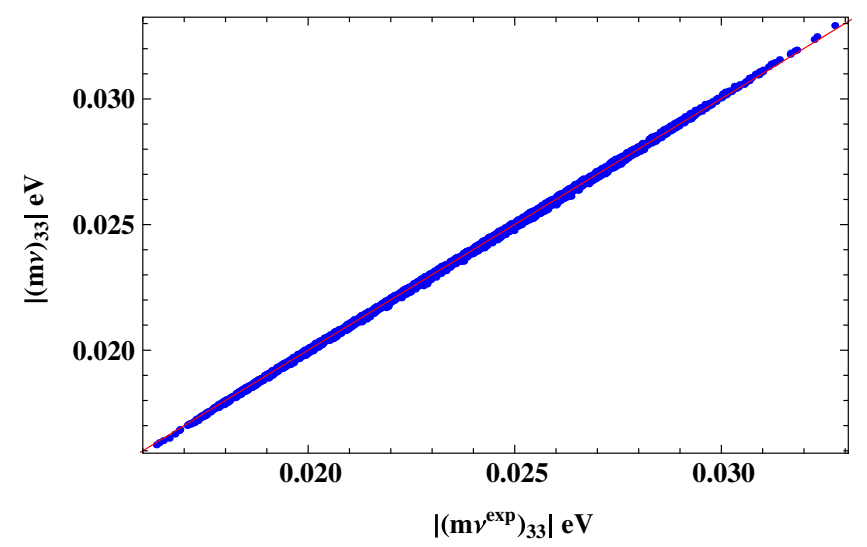

FIG. 2. Allowed region between $\left|\left(m_{\nu}\right)_{33}^{\exp }\right|$ and $\left|\left(m_{\nu}\right)_{33}\right|$, where the red line represents $\left|\left(m_{\nu}\right)_{33}^{\exp }\right|=\left|\left(m_{\nu}\right)_{33}\right|$.

$$
m_{\nu} \approx\left[\begin{array}{ccc}
a & 0 & a r_{31} \\
0 & b & b r_{42} \\
a r_{31} & b r_{42} & a r_{31}^{2}+b r_{42}^{2}
\end{array}\right]
$$

It implies that $\left(m_{\nu}\right)_{33}$ component is uniquely fixed once $a$, $b, r_{31}, r_{42}$ are determined by experimental values. While experimental value of $\left(m_{\nu}\right)_{33} ;\left(\left(m_{\nu}^{\exp }\right)_{33} \equiv\right)\left[U_{\mathrm{PMNS}}^{*} \operatorname{diag}\left(m_{1}\right.\right.$, $\left.\left.m_{2}, m_{3}\right) U_{\mathrm{PMNS}}^{\dagger}\right]_{33}$, is independently determined by experimental result, too. In Fig. 2, we show the allowed region between $\left|\left(m_{\nu}\right)_{33}^{\exp }\right|$ and $\left|\left(m_{\nu}\right)_{33}\right|$, where the red line represents $\left|\left(m_{\nu}\right)_{33}^{\exp }\right|=\left|\left(m_{\nu}\right)_{33}\right|$. It suggests the theoretical consequence is in favor of the experimental result that is consistent with the original paper [4].

\section{B. Phenomenology}

In this subsection, we discuss phenomenology of the model such as collider physics and dark matter physics.
At the LHC $Z^{\prime}$ can be produced as it couples to the SM quarks, and can decay into the SM leptons providing clear di-lepton signal. On the other hand the signatures from exotic scalar bosons are more complicated containing more particles in final states and their branching ratios depend on the parameters in the scalar potential so that we have less predictability, although they can also be produced via $Z^{\prime}$ interaction and through electroweak interaction if an exotic scalar boson comes from iso-doublet. Thus, we focus on $Z^{\prime}$ production in $s$-channel followed by decay mode of $Z^{\prime} \rightarrow \ell^{+} \ell^{-}$and estimate the constraints for new gauge coupling constant and mass of $Z^{\prime}$. Then dark matter relic density is briefly discussed taking into account the constraint for $Z^{\prime}$ interaction.

\section{Collider physics and constraints}

Here, we explore collider physics focusing on $Z^{\prime}$ boson and provide constraints for its mass and gauge coupling constant. The relevant gauge interactions are given by

$$
\begin{aligned}
\mathcal{L}_{\mathrm{int}}= & g_{B L} Z_{\mu}^{\prime}\left[\frac{1}{3} \bar{Q}_{L} \gamma^{\mu} Q_{L}+\frac{1}{3} \bar{u}_{R} \gamma^{\mu} u_{R}+\frac{1}{3} \bar{d}_{R} \gamma^{\mu} d_{R}\right. \\
& -\bar{L} \gamma^{\mu} L-\bar{e}_{R} \gamma^{\mu} e_{R}+\frac{1}{2} Q_{N_{R_{i}}}^{B-L} \bar{N}_{R_{i}} \gamma^{\mu} \gamma^{5} N_{R_{i}} \\
& \left.+Q_{\Phi}^{B-L}\left(\partial^{\mu} \Phi^{*} \Phi-\Phi^{*} \partial^{\mu} \Phi\right)\right]
\end{aligned}
$$

where flavor indices for the SM fermions are omitted and $\Phi=\left\{\eta_{1}, \eta_{1^{\prime}}, \eta_{D}, \varphi_{8}, \varphi_{8}^{\prime}, \varphi_{10}, \zeta\right\}$; note that $\varphi_{2}$ is not included here since we assume its $C P$-odd component is Nambu-Goldstone boson absorbed by $Z^{\prime}$. The mass of $Z^{\prime}$ is given by $m_{Z^{\prime}}=g_{B L} \sqrt{\sum_{\Phi_{B L}}\left(Q_{\Phi}^{B-L} v_{\Phi_{B L}}\right)^{2}}$ where $\Phi_{B L}$ and $v_{\Phi_{B L}}$ indicate scalar field with $B-L$ charge $Q_{\Phi}^{B-L}$ and its $\mathrm{VEV}$, respectively. The partial decay widths of $Z^{\prime}$ are estimated as

$$
\begin{aligned}
\Gamma_{Z^{\prime} \rightarrow \bar{f}_{\mathrm{SM}} f_{\mathrm{SM}}}= & \frac{\left(Q^{B-L} g_{B L}\right)^{2}}{12 \pi} m_{Z^{\prime}}\left(1-\frac{4 m_{f_{\mathrm{SM}}}^{2}}{m_{Z^{\prime}}^{2}}\right)^{\frac{3}{2}}, \\
\Gamma_{Z^{\prime} \rightarrow \bar{N}_{R_{i}} N_{R_{i}}}= & \frac{\left(Q_{N_{R_{i}}}^{B-L} g_{B L}\right)^{2}}{96 \pi}\left(1-\frac{4 m_{N_{R_{i}}}^{2}}{m_{Z^{\prime}}^{2}}\right)^{\frac{3}{2}}, \\
\Gamma_{Z^{\prime} \rightarrow \Phi_{1} \Phi_{2}}= & \frac{\left(Q_{\Phi}^{B-L} g_{B L}\right)^{2}}{48 \pi} m_{Z^{\prime}} \lambda^{\frac{1}{2}}\left(m_{Z^{\prime}}, m_{\Phi_{1}}, m_{\Phi_{2}}\right) \\
& \times\left[1-\frac{2\left(m_{\Phi_{1}}^{2}+m_{\Phi_{2}}^{2}\right)}{m_{Z^{\prime}}^{2}}+\frac{\left(m_{\Phi_{1}}^{2}-m_{\phi_{2}}^{2}\right)}{m_{Z^{\prime}}^{4}}\right], \\
\lambda\left(m_{Z^{\prime}}, m_{\Phi_{1}}, m_{\Phi_{2}}\right)= & 1+\frac{m_{\Phi_{1}}^{4}}{m_{Z^{\prime}}^{4}}+\frac{m_{\Phi_{2}}^{4}}{m_{Z^{\prime}}^{4}}-2 \frac{m_{\Phi_{1}}^{2}}{m_{Z^{\prime}}^{4}} \\
& -2 \frac{m_{\Phi_{1}}^{2}}{m_{Z^{\prime}}^{2}}-2 \frac{m_{\Phi_{2}}^{2}}{m_{Z^{\prime}}^{2}},
\end{aligned}
$$


TABLE III. Branching ratios for $Z^{\prime}$ decay in cases: (1) $m_{Z^{\prime}}<2 m_{N_{R_{i}}}$ and $m_{Z^{\prime}}<2 m_{\Phi}$; (2) $m_{Z^{\prime}}>2 m_{N_{R_{i}}}$ and $m_{Z^{\prime}}<2 m_{\Phi}$; (3) $m_{Z^{\prime}}>2 m_{N_{R_{i}}}$ and $m_{Z^{\prime}}>2 m_{\Phi}$ where we ignored dependence on final state mass assuming $m_{N_{R_{i}}, \Phi}^{2} \ll m_{Z^{\prime}}^{2}$ if kinematically allowed in case (2) and (3). For exotic scalar modes, BRs for all components are summed up.

\begin{tabular}{llccccccccccc}
\hline \hline & $\ell_{i}^{+} \ell_{i}^{-}$ & $\bar{\nu}_{i} \nu_{i}$ & $\bar{q}_{i} q_{i}$ & $N_{R_{1,2}}$ & $N_{R_{3}}$ & $\eta_{1}^{*} \eta_{1}$ & $\eta_{1^{\prime}}^{*} \eta_{1^{\prime}}$ & $\eta_{D}^{*} \eta_{D}$ & $\varphi_{8}^{*} \varphi_{8}$ & $\varphi_{8}^{\prime *} \varphi_{8}^{\prime}$ & $\varphi_{10}^{*} \varphi_{10}$ & $\zeta^{*} \zeta$ \\
\hline Case (1) & 0.15 & 0.077 & 0.051 & 0.0 & 0.0 & 0.0 & 0.0 & 0.0 & 0.0 & 0.0 & 0.0 & 0.0 \\
Case (2) & 0.073 & 0.037 & 0.024 & 0.15 & 0.23 & 0.0 & 0.0 & 0.0 & 0.0 & 0.0 & 0.0 & 0.0 \\
Case (3) & 0.0076 & 0.0038 & 0.0025 & 0.015 & 0.024 & 0.034 & 0.034 & 0.068 & 0.12 & 0.12 & 0.19 & 0.068 \\
\hline \hline
\end{tabular}

where $f_{\mathrm{SM}}$ denotes the SM fermions and $\left\{\Phi_{1}, \Phi_{2}\right\}$ indicate components of $\Phi$. We estimate branching ratios (BRs) for $Z^{\prime}$ decay in cases: (1) $m_{Z^{\prime}}<2 m_{N_{R_{i}}}$ and $m_{Z^{\prime}}<2 m_{\Phi}$; (2) $m_{Z^{\prime}}>2 m_{N_{R_{i}}}$ and $m_{Z^{\prime}}<2 m_{\Phi}$; (3) $m_{Z^{\prime}}>2 m_{N_{R_{i}}}$ and $m_{Z^{\prime}}>2 m_{\Phi}$, where $m_{\Phi}$ represents exotic scalar mass assuming they are mostly the same scale. In Table III, we show the BRs for $Z^{\prime}$ decay where we ignored dependence on final state mass assuming $m_{N_{R_{i}},}^{2}, \ll m_{Z^{\prime}}^{2}$ if kinematically allowed in cases (2) and (3) for simplicity. We find that BRs for the SM fermions are significantly suppressed when all exotic scalar modes are open.

Then we discuss constraint on $g_{B L}$ from the LHC experiments for three cases above. Our $Z^{\prime}$ boson is produced via $Z^{\prime} \bar{q} q$ coupling and the production cross section is estimated using CalcHEP 3.6 [35] implementing relevant interactions. The most stringent constraint comes from the process $p p \rightarrow Z^{\prime} \rightarrow \ell^{+} \ell^{-}(\ell=e, \mu)$ and we estimate the corresponding cross section for each case. In Fig. 3, we compare ratio between $\sigma \cdot \operatorname{BR}\left(p p \rightarrow Z^{\prime} \rightarrow\right.$ $\left.\ell^{+} \ell^{-}\right)$and $\sigma \cdot \operatorname{BR}\left(p p \rightarrow Z \rightarrow \ell^{+} \ell^{-}\right)$in our model with the experimental constraints corresponding to $95 \%$ confidence level (CL) observed limit indicated by red curve [36] where solid, dashed and dotted curve correspond to cases (1), (2), and (3), respectively, and we apply $g_{B L}=$ $0.3(0.1)$ in left(right) plots. Thus, the lower limit of mass $Z^{\prime}$ is relaxed when the exotic scalar modes of $Z^{\prime}$ decay are kinematically allowed: the lower limit of $m_{Z^{\prime}}$ is around $3300(2000) \mathrm{GeV}$ for $g_{B L}=0.3(0.1)$ in $95 \% \mathrm{CL}$. For case (3), the $Z^{\prime}$ boson dominantly decays into exotic scalar bosons which further decay into SM particles via gauge interaction and/or couplings in the scalar potential providing multi-particle final states. The detailed analysis of the scalar modes is beyond the scope of our analysis.

\section{Dark matter}

In this subsection, we discuss a dark matter candidate; $X_{R} \equiv N_{R_{3}}$, whose stability is assured by the $U(1)_{B-L}$ symmetry with alternative charge assignment for the SM singlet fermions. Here, let us assume any contributions from the Higgs mediating interaction are negligibly small so as to avoid the constraints from direct detection searches as LUX [37], XENON1T [38], and PandaX-II [39]. Then DM annihilation processes are dominated by the gauge interaction with $Z^{\prime}$ and GB $\alpha_{G} \equiv z_{\varphi_{10}}$ mainly originated from $\varphi_{10}$, and their relevant Lagrangian in basis of mass eigenstate is found to be
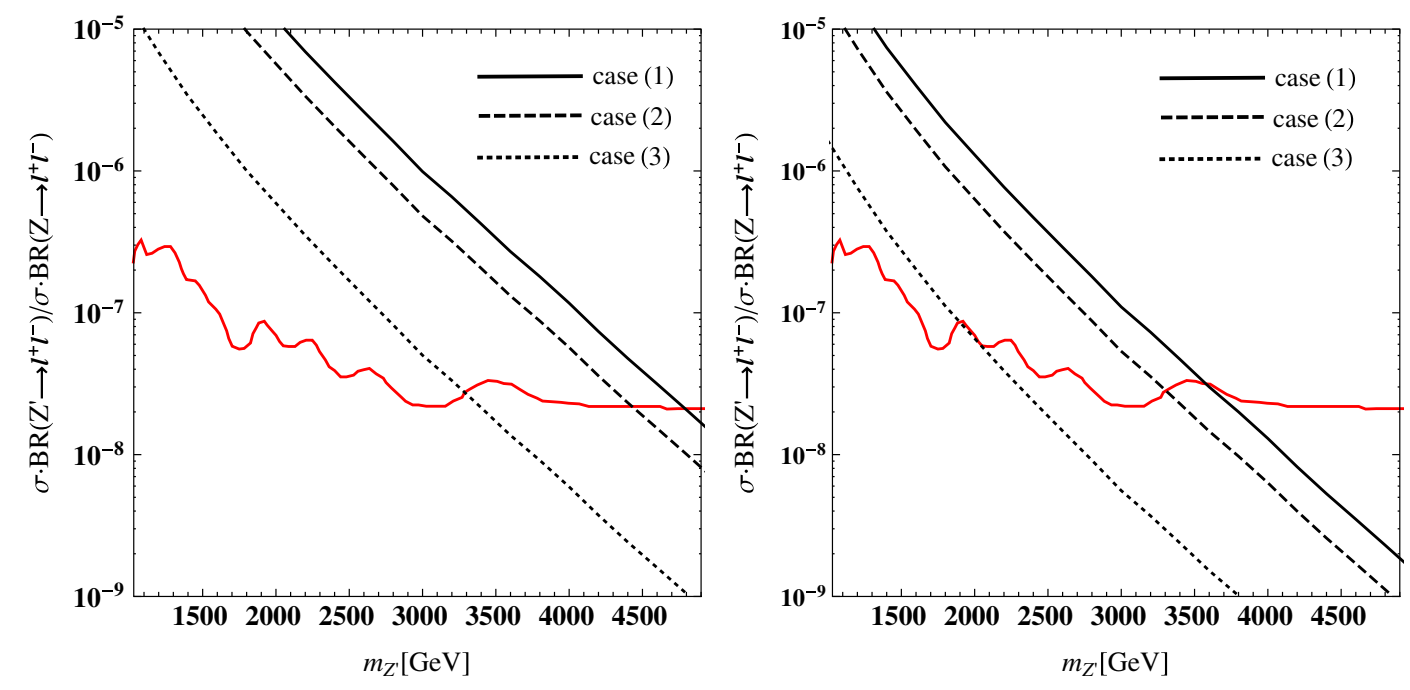

FIG. 3. The ratio between $\sigma \cdot \operatorname{BR}\left(p p \rightarrow Z^{\prime} \rightarrow \ell^{+} \ell^{-}\right)$and $\sigma \cdot \operatorname{BR}\left(p p \rightarrow Z \rightarrow \ell^{+} \ell^{-}\right)$where $\ell=e, \mu$ and the red curve indicates the experimental constraints which corresponds to $95 \%$ confidence level observed limit. The left(right) plot corresponds to $g_{B L}=0.3(0.1)$. 


$$
-\mathcal{L} \supset \frac{1}{2} Q_{B L}^{X} g_{B L} \bar{X} \gamma^{\mu} \gamma_{5} X Z_{\mu}^{\prime}+i \frac{M_{X}}{v_{\varphi_{10}}} \bar{X} P_{R} X \alpha_{G}+\text { c.c. }
$$

where $Q_{B L}^{X}=5, M_{X} \equiv y_{N_{3}} v_{\varphi_{10}} / \sqrt{2}, v_{\varphi_{10}} \ll v_{\varphi_{2}}$. Here, we require $Z^{\prime}$ mass and gauge coupling $g_{B L}$ to satisfy the relation $g_{B L} / m_{Z^{\prime}} \lesssim 1 /(6.9 \mathrm{TeV})$ from LEP experiment [40] as well as the constraints from the LHC experiments as discussed in the previous subsection. The relic density of DM is then given by [41,42]

$$
\Omega h^{2} \approx \frac{1.07 \times 10^{9}}{\sqrt{g_{*}\left(x_{f}\right)} M_{\mathrm{Pl}} J\left(x_{f}\right)[\mathrm{GeV}]},
$$

where $g^{*}\left(x_{f} \approx 25\right)$ is the degrees of freedom for relativistic particles at temperature $T_{f}=M_{X} / x_{f}, M_{\mathrm{Pl}} \approx 1.22 \times 10^{19} \mathrm{GeV}$, and $J\left(x_{f}\right)\left(\equiv \int_{x_{f}}^{\infty} d x \frac{\left\langle\sigma v_{\text {rer }}\right\rangle}{x^{2}}\right)$ is given by $[32,43]$

$$
\begin{gathered}
J\left(x_{f}\right)=\int_{x_{f}}^{\infty} d x\left[\frac{\int_{4 M_{X}^{2}}^{\infty} d s \sqrt{s-4 M_{X}^{2}}\left[W_{Z^{\prime}}(s)+W_{z_{\phi^{\prime}}}(s)\right] K_{1}\left(\frac{\sqrt{s}}{M_{X}} x\right)}{16 M_{X}^{5} x\left[K_{2}(x)\right]^{2}}\right], \\
W_{Z^{\prime}}(s) \approx \frac{4\left(s-4 M_{X}^{2}\right)}{3 \pi}\left|\frac{5 g_{B L}^{2}}{s-m_{Z^{\prime}}^{2}+i m_{Z^{\prime}} \Gamma_{Z^{\prime}}}\right|^{2} \sum_{f} \sqrt{1-\frac{4 m_{f}^{2}}{s}\left(s+2 m_{f}^{2}\right)\left|Q_{B L}^{f}\right|^{2},} \\
W_{\alpha_{G}}(s) \simeq \frac{\left|M_{X}\right|^{4}}{64 \pi v_{\varphi_{10}}^{4}}\left[\left(3 s^{2}-4 M_{X}^{4}\right)\left(\frac{\pi}{2 s M_{X}^{2}} \sqrt{\frac{M_{X}^{4}}{4 s M_{X}^{2}-s^{2}}}-\frac{\tan ^{-1}\left[\frac{s-2 M_{X}^{2}}{\sqrt{s\left(4 M_{X}^{2}-s\right)}}\right]}{s^{3 / 2} \sqrt{4 M_{X}^{2}-s}}\right)-4\right],
\end{gathered}
$$

where we assumed $Z^{\prime}$ boson and scalar bosons are heavier than $X$ to forbid corresponding annihilation processes kinematically, for simplicity. Here, decay width of $Z^{\prime}$ is given by Eq. (4.12) where $Z^{\prime}$ can decay into $2 X$, if kinematically allowed. We find that two characterized solutions of measured relic density $\Omega h^{2} \approx 0.12$ [44] in the above formula. The first one is a sharp region at around $M_{X} \sim m_{Z^{\prime}} / 2$, that is a resonant solution from the contribution $2 X \rightarrow Z^{\prime} \rightarrow f \bar{f}$ in Eq. (4.16). The second one is the region in lighter mass of DM that mainly arises from the contribution $2 X \rightarrow 2 \alpha_{G}$ in Eq. (4.17). In the former case $\mathrm{DM}$ mass is around $\mathrm{TeV}$ scale to obtain right relic density due to the collider constraints for $Z^{\prime}$ mass while in the latter case DM mass can be $\mathcal{O}(10) \mathrm{GeV}$ to $\mathcal{O}(100) \mathrm{GeV}$ which depend on the coupling factor $M_{X} / v_{\varphi_{10}}$; for more details, see, e.g., Refs. [32,43].

\section{CONCLUSION}

We have systematically explored the origins of neutrino textures in the canonical seesaw model with two right-handed neutrinos based on global $\mathrm{U}(1)_{\mu-\tau}$ flavor symmetry, and smaller non-Abelian flavor symmetries, and we have shown several promising symmetries to find predictive textures, $U(1)_{\mu-\tau}$ and $D_{4}$, depending on appropriate charge assignments of our fields. Moreover, we have found that $D_{4}$ symmetry can realize a predictive texture $b$ only. Then we have proposed a concrete model based on local $U(1)_{B-L}$ and $D_{4}$ symmetries that involves a dark matter candidate and extra gauge boson. To show properties of the model, we have analyzed the neutrino physics, collider physics regarding $Z^{\prime}$ boson and relic density of dark matter. We have shown that constraints for $Z^{\prime}$ mass and interactions can be relaxed when exotic scalar modes of $Z^{\prime}$ decay are kinematically open, and relic density of dark matter can be explained by annihilation mode via $Z^{\prime}$ exchange and/or annihilation into physical Goldstone bosons.

\section{ACKNOWLEDGMENTS}

T. K. was is supported in part by MEXT KAKENHI Grant No. JP17H05395. This research is supported by the Ministry of Science, ICT and Future Planning, Gyeongsangbuk-do and Pohang City (H.O.). H. O. is sincerely grateful for the KIAS member.

\section{APPENDIX: MULTIPLICATION RULES FOR $D_{\mathbf{4}}$ GROUP}

Here, we show the valid multiplication rules for $D_{4}$ group that consists of four irreducible singlets $1,1^{\prime}, 1^{\prime \prime}, 1^{\prime \prime \prime}$ and one irreducible doublet 2, where we have used a real representation [45]; 


$$
\left[\begin{array}{l}
x_{1} \\
x_{2}
\end{array}\right]_{2} \otimes\left[\begin{array}{l}
y_{1} \\
y_{2}
\end{array}\right]_{2}=\left(x_{1} y_{1}+x_{2} y_{2}\right)_{1} \oplus\left(x_{1} y_{1}-x_{2} y_{2}\right)_{1^{\prime}} \oplus\left(x_{1} y_{2}+x_{2} y_{1}\right)_{1^{\prime \prime}} \oplus\left(x_{1} y_{2}-x_{2} y_{1}\right)_{1^{\prime \prime \prime}}
$$

The other relations are given by $2 \otimes 1\left(1^{\prime}, 1^{\prime \prime}, 1^{\prime \prime \prime}\right)=2,1^{\prime} \otimes 1^{\prime}\left(1^{\prime \prime}, 1^{\prime \prime \prime}\right)=1\left(1^{\prime \prime \prime}, 1^{\prime \prime}\right), 1^{\prime \prime} \otimes 1^{\prime \prime}\left(1^{\prime \prime \prime}\right)=1\left(1^{\prime}\right)$, and, $1^{\prime \prime \prime} \otimes 1^{\prime \prime \prime}=1$ in Ref. [7].

[1] H. Fritzsch and Z.z. Xing, Prog. Part. Nucl. Phys. 45, 1 (2000).

[2] D. V. Forero, M. Tortola, and J. W. F. Valle, Phys. Rev. D 90, 093006 (2014).

[3] H. Fritzsch, Z. z. Xing, and S. Zhou, J. High Energy Phys. 09 (2011) 083.

[4] D. M. Barreiros, R. G. Felipe, and F. R. Joaquim, Phys. Rev. D 97, 115016 (2018).

[5] T. Rink and K. Schmitz, J. High Energy Phys. 03 (2017) 158.

[6] G. Altarelli and F. Feruglio, Rev. Mod. Phys. 82, 2701 (2010).

[7] H. Ishimori, T. Kobayashi, H. Ohki, Y. Shimizu, H. Okada, and M. Tanimoto, Prog. Theor. Phys. Suppl. 183, 1 (2010); Lect. Notes Phys. 858, 1 (2012).

[8] S. F. King and C. Luhn, Rep. Prog. Phys. 76, 056201 (2013).

[9] T. Kobayashi, H. P. Nilles, F. Ploger, S. Raby, and M. Ratz, Nucl. Phys. B768, 135 (2007).

[10] T. Kobayashi, S. Raby, and R. J. Zhang, Nucl. Phys. B704, 3 (2005).

[11] P. Ko, T. Kobayashi, J. h. Park, and S. Raby, Phys. Rev. D 76, 035005 (2007); 76, 059901(E) (2007).

[12] F. Beye, T. Kobayashi, and S. Kuwakino, Phys. Lett. B 736, 433 (2014).

[13] H. Abe, K. S. Choi, T. Kobayashi, and H. Ohki, Nucl. Phys. B820, 317 (2009).

[14] M. Berasaluce-Gonzalez, P. G. Camara, F. Marchesano, D. Regalado, and A. M. Uranga, J. High Energy Phys. 09 (2012) 059.

[15] T. Kobayashi, S. Nagamoto, S. Takada, S. Tamba, and T. H. Tatsuishi, Phys. Rev. D 97, 116002 (2018).

[16] W. Grimus and L. Lavoura, Phys. Lett. B 572, 189 (2003).

[17] W. Grimus, A. S. Joshipura, S. Kaneko, L. Lavoura, and M. Tanimoto, J. High Energy Phys. 07 (2004) 078.

[18] H. Ishimori, T. Kobayashi, H. Ohki, Y. Omura, R. Takahashi, and M. Tanimoto, Phys. Lett. B 662, 178 (2008); Phys. Rev. D 77, 115005 (2008).

[19] A. Adulpravitchai, A. Blum, and C. Hagedorn, J. High Energy Phys. 03 (2009) 046.

[20] C. Hagedorn and R. Ziegler, Phys. Rev. D 82, 053011 (2010).
[21] D. Meloni, S. Morisi, and E. Peinado, Phys. Lett. B 703, 281 (2011).

[22] T. Araki, J. Heeck, and J. Kubo, J. High Energy Phys. 07 (2012) 083.

[23] J.C. Gomez-Izquierdo and M. Mondragon, arXiv:1804 .08746

[24] J. C. Gomez-Izquierdo, Eur. Phys. J. C 77, 551 (2017).

[25] H. Abe, K. S. Choi, T. Kobayashi, and H. Ohki, Phys. Rev. D 80, 126006 (2009); 81, 126003 (2010).

[26] F. Marchesano, D. Regalado, and L. Vazquez-Mercado, J. High Energy Phys. 09 (2013) 028.

[27] H. Abe, T. Kobayashi, H. Ohki, K. Sumita, and Y. Tatsuta, J. High Energy Phys. 06 (2014) 017.

[28] J. C. Montero and V. Pleitez, Phys. Lett. B 675, 64 (2009).

[29] E. Ma and R. Srivastava, Phys. Lett. B 741, 217 (2015).

[30] S. Singirala, R. Mohanta, and S. Patra, arXiv:1704.01107.

[31] T. Nomura and H. Okada, arXiv:1705.08309.

[32] T. Nomura and H. Okada, Eur. Phys. J. C 78, 189 (2018).

[33] T. Nomura and H. Okada, Phys. Lett. B 781, 561 (2018).

[34] C. Q. Geng and H. Okada, Phys. Dark Universe 20, 13 (2018).

[35] A. Belyaev, N. D. Christensen, and A. Pukhov, Comput. Phys. Commun. 184, 1729 (2013).

[36] (CMS Collaboration), CERN LHC Report No. CMS-PASEXO-18-006.

[37] D. S. Akerib et al. (LUX Collaboration), Phys. Rev. Lett. 118, 021303 (2017).

[38] E. Aprile et al. (XENON Collaboration), arXiv:1705.06655.

[39] X. Cui et al. (PandaX-II Collaboration), Phys. Rev. Lett. 119, 181302 (2017).

[40] S. Schael et al. (ALEPH and DELPHI and L3 and OPAL and LEP Electroweak Collaborations), Phys. Rep. 532, 119 (2013).

[41] K. Griest and D. Seckel, Phys. Rev. D 43, 3191 (1991).

[42] J. Edsjo and P. Gondolo, Phys. Rev. D 56, 1879 (1997).

[43] T. Nomura and H. Okada, Phys. Rev. D 97, 075038 (2018).

[44] P. A. R. Ade et al. (Planck Collaboration), Astron. Astrophys. 571, A16 (2014).

[45] V. V. Vien and H. N. Long, Int. J. Mod. Phys. A 28, 1350159 (2013). 